\title{
Implementation of PAR-CLIP to characterize RNA-protein \\ interactions in prokaryotes at nucleotide resolution
}

\section{Sandeep Ojha ${ }^{1}$ and Chaitanya Jain ${ }^{1 *}$}

${ }^{1}$ Department of Biochemistry and Molecular Biology, University of Miami Miller School of Medicine, Miami, FL 33136, USA. Tel: (305) 243-7229, Fax: (305) 243-3955, e-mail: cjain@miami.edu.

${ }^{*}$ Corresponding author 


\section{Abstract}

The identification of RNAs that are recognized by RNA-binding proteins (RNA-BPs) using techniques such as "Crosslinking and Immunoprecipitation" (CLIP) has revolutionized the genome-wide discovery of RNA-BP RNA targets. Among the different versions of CLIP that have been developed, the use of photoactivable nucleoside analogs incorporated into cellular RNA has resulted in high efficiency photoactivable ribonucleoside-enhanced CLIP (PAR-CLIP). Nonetheless, PAR-CLIP has not yet been applied in prokaryotes. To determine if PAR-CLIP can be used in prokaryotes, we determined suitable conditions for the incorporation of 4-thiouridine (4SU), a photoactivable nucleoside, into E. coli RNA, and for the isolation of crosslinked RNA. Applying this technique to Hfq, a well-characterized regulator of small RNA (sRNA)-messenger RNA (mRNA) interactions, we showed that PAR-CLIP identified most of the known sRNA targets of $\mathrm{Hfq}$, as well as functionally relevant sites of Hfq-mRNA interactions at nucleotide resolution. Based on our findings, PAR-CLIP represents an improved method to identify both the RNAs and the specific regulatory sites that are recognized by RNA-BPs in prokaryotes.

\section{Introduction}

RNA-BPs constitute a large and diverse class of proteins that regulate nearly every aspect of RNA function inside the cell. Among the hundreds of such proteins, the specific functions of many are not well understood. An important goal towards defining the cellular roles of RNA-BPs is to identify the RNAs they interact with inside the cell - not only to better understand the biological functions of the RNA-BPs - but also the basis of RNA recognition through a systematic analysis of their RNA targets.

The first genome-wide method developed to identify the RNAs recognized by RNA-BPs was based on RNA immunoprecipitation (RIP) (1). In this method, the protein of interest, as well 
as its associated RNAs, are purified by using anti-RNA-BP antibodies. Thereafter, the RNAs are extracted and their identities are revealed either by sequencing or by hybridization to DNA microarrays. Over the past few years RIP has become largely supplanted by CLIP, a method that is conceptually similar to RIP, except that the RNA-BPs are first crosslinked to their RNA targets in vivo $(2,3)$. The development of CLIP stemmed, in part, from concerns that the noncovalent nature of protein-RNA interactions in RIP could result in the loss of many bound RNAs, especially if extensive purification steps were employed.

Two different procedures for crosslinking have been applied for CLIP. In the first, a chemical crosslinker, such as formaldehyde, has been used $(4,5)$. In the second approach, RNA-protein crosslinks are generated by irradiating cells with short-wavelength ultraviolet (UV) light $(2,3)$. Despite widespread adoption, each method suffers from limitations that can reduce their effectiveness. With chemical crosslinking, a significant drawback is that the crosslinking reagents induce not only protein-RNA, but also protein-DNA and protein-protein crosslinks. Therefore, the RNAs identified can include those that are recognized by proteins that interact with the RNA-BP of interest. In contrast, the efficiency of crosslinking with standard UV-CLIP is low, which can impact the coverage of RNA targets identified using this method. Any improvements to these techniques, therefore, are expected to yield a more complete and accurate picture of RNA-BP targets inside the cell.

To improve on existing procedures, a new method, PAR-CLIP, was developed (6). In this method, cells are grown in the presence of a photoactivable nucleotide added to the growth medium, the most commonly used being $4 S U$. Incorporation of $4 S U$ into RNA, followed by irradiation with long-wavelength UV light, has been reported to enhance crosslinking efficiency by $100-1000$-fold relative to conventional CLIP (6). An additional benefit of PAR-CLIP is that crosslinked $4 \mathrm{SU}$ residues are misread as cytosine residues with high frequency during the reverse transcription step of sequence library preparation (6-8). Consequently, an analysis of T 
to $\mathrm{C}$ conversion frequency in the sequence data directly identifies the sites of cross-linking in the RNAs.

Although PAR-CLIP is now established in eukaryotes, to the best of our knowledge, it has not yet been implemented in prokaryotes. To determine the feasibility of this technique for prokaryotic organisms, we first identified suitable conditions for its use in E. coli. We then applied PAR-CLIP to Hfq, a well-characterized regulator of sRNA-mRNA interactions, and found that PAR-CLIP effectively identifies most of the sRNAs previously shown to be associated with Hfq. Through the use of RNA-Seq and quantitative proteomics, we further identified a number of mRNAs that were both regulated by $\mathrm{Hfq}$ and contained PAR-CLIP cross-linking sites. Significantly, we found that mutation of residues in the vicinity of the cross-linking sites reduced the extent of Hfq-mediated regulation in almost every case that was analyzed. We anticipate that the power of PAR-CLIP to identify biologically relevant cross-linking sites will be highly useful in the future to define the function of RNA-BPs inside the cell.

\section{Results}

Incorporation of $4 S U$ into E. coli RNA

To determine whether 4SU can be incorporated into E. coli RNA, a wild-type strain (CJ2109) was grown in LB medium or in LB supplemented with $300 \mu \mathrm{M} 4 \mathrm{SU}$. RNA was isolated from mid-log cultures and digested with nucleases and phosphatases to nucleosides. The digested RNA was analyzed by using high performance liquid chromatography (HPLC) with readings at $260 \mathrm{~nm}$ (all nucleotides) or $330 \mathrm{~nm}$ (4SU). Both sets of RNAs gave similar peaks for the standard nucleosides at $260 \mathrm{nM}$ (Fig. 1). However, with detection at $330 \mathrm{~nm}$, the RNA samples isolated from cells grown with $4 \mathrm{SU}$ yielded a significantly higher signal compared to cells without 4SU. Based on peak heights and a comparison with defined nucleoside standards, 
we calculated that $1.3 \%$ of uridine residues in the latter RNA sample were replaced with 4 SU. These experiments indicated that $4 \mathrm{SU}$ can be incorporated into RNA in vivo.

\section{PAR-CLIP on FLAG-tagged proteins}

To determine whether 4SU containing RNA can be crosslinked to RNA-BPs, CJ2109 was transformed with pCJ1099, a plasmid that encodes the DEAD-box protein RhIE tagged with a 3X FLAG epitope at its C-terminal end. We have previously shown that RhIE binds to the 50S ribosomal subunit and to $70 \mathrm{~S}$ ribosomes (9), and therefore, is likely an RNA-binding protein. The transformed strain (CJ2191) was grown to midlog phase in LB medium in the absence or the presence of different concentrations of $4 \mathrm{SU}$ in the medium, followed by harvesting of cells and irradiation with long-wavelength UV light $(365 \mathrm{~nm})$. The cells were lysed, incubated with beads that were coupled to anti-FLAG antibodies, and unbound proteins were removed through multiple washes. Thereafter, the crosslinked RNAs were fragmented on beads and labeled at the $5^{\prime}$ end with ${ }^{32} \mathrm{P}$. The labeled proteins were eluted from the beads, fractionated by using polyacrylamide gel electrophoresis (PAGE), transferred to nitrocellulose, and exposed to a phosphorimager screen to visualize the crosslinked RNAs.

A comparison of the signal derived from cells grown in the presence of different amounts of $4 \mathrm{SU}$ indicated that low levels of radiolabeled RNA were crosslinked to RhIE when cells were grown in the absence or the presence of $100 \mu \mathrm{M}$ 4SU (Fig. 2A). Significant amounts of crosslinked signal was observed for cells grown with $250 \mu \mathrm{M} 4 \mathrm{SU}$ and a further signal increase was observed with $500 \mu \mathrm{M}$ 4SU. These results show that 4SU-containing RNAs can be crosslinked to proteins. Surprisingly, when cells were grown with $1 \mathrm{mM} 4 \mathrm{SU}$, the signal was found to be reduced. However, we noticed those cells exhibited slower growth, suggesting that the reduced efficiency of $4 \mathrm{SU}$ incorporation into RNA in this instance could be related to growth inhibition. 
To optimize the crosslinking efficiency of 4 SU-containing RNAs, cells were irradiated with $365 \mathrm{~nm}$ UV light for different amounts of time (Fig. 2B). Increasing the irradiation time for up to 10 minutes resulted in increased levels of crosslinking, indicating that the extent of crosslinking was not saturated even with the longest irradiation time used.

We were next interested in determining whether the efficacy UV irradiation could be limited by the medium used for growth. We compared the absorbance of LB medium, which is commonly used to grow $E$. coli cells, with a rich-defined medium (RDM). RDM, unlike LB, is a clear solution, and might be expected to absorb less long-wavelength UV light. We found that RDM was virtually transparent to $365 \mathrm{~nm}$ UV light, whereas LB medium displayed an absorbance of 0.6 (Fig. 2C), which corresponds to $75 \%$ absorbance of UV light by $1 \mathrm{~cm}$ of medium. As expected, the addition of $250 \mu \mathrm{M}$ of $4 \mathrm{SU}$ increased absorbance in both growth media. Because the standard CLIP protocol uses $254 \mathrm{~nm}$ UV light, we also measured the absorbance of both LB and RDM at $254 \mathrm{~nm}$. In each case, the absorbance was 10 or greater, likely because of the presence of nucleotides or their analogs in the media, which implies that when standard crosslinking is performed using $254 \mathrm{~nm} \mathrm{UV}$, the effective depth to which the UV light will penetrate is less than $1 \mathrm{~mm}$. Although several factors determine the overall efficiency of cross-linking, the inability of $254 \mathrm{~nm}$ UV to penetrate the growth media might be considered a significant drawback of using such short wavelength light for cross-linking purposes.

To verify that absorbance by LB could be reducing cross-linking efficiency, CJ2191 was growth in LB/4SU and either cross-linked directly after cell harvest, or after pelleting the cells and resuspending them in saline $(0.85 \% \mathrm{NaCl})$. Consistent with the results of Fig. $2 \mathrm{C}$, we found that washing off the growth medium significantly increased the cross-linked signal (Fig. 2D). Nonetheless, given that many E. coli RNAs have short half-lives, and since a washing step would increase the time between harvesting cultures and cross-linking, we decided to adopt RDM as the growth medium of choice for our studies. 
Because we had observed a reduction in cross-linking signal when cells were grown with $1000 \mu \mathrm{M}$ of $4 \mathrm{SU}$ (Fig. 2A), we measured the effect of different concentrations of $4 \mathrm{SU}$ on cell growth. The addition of $4 S U$ up to $250 \mu \mathrm{M}$ to RDM had no significant effect on the growth rate, but increased $4 \mathrm{SU}$ concentrations was found to increase cell doubling time, indicating growth inhibition (Fig. 2E). In order to perform CLIP without perturbing growth, the downstream studies were performed using $250 \mu \mathrm{M}$ of $4 \mathrm{SU}$ in the growth medium.

Finally, with an intention to generate CLIP data using a model RNA-BP that is expressed at physiological levels, we constructed a strain (CJ2192) in which the chromosomal locus expressing Hfq RNA-BP was modified to append a 3X FLAG-epitope at its C-terminus. Hfq is a well-studied regulator of gene expression, and multiple studies have shown that it binds to several regulatory sRNAs inside the cell and facilitates sRNA-mRNA interactions $(10,11)$. CJ2192 was grown in the presence of 4SU, and the cells were crosslinked at $365 \mathrm{~nm}$. To determine whether there is any non-specific background, the same procedure was also performed on the untagged strain, CJ2109. After crosslinking, the RNAs were analyzed by FLAG-tag protein purification and gel electrophoresis, as described above. With CJ2192, a strong signal consistent with the migration of Hfq could be observed (Fig. 2F). In contrast, very little signal was observed with the untagged strain, which could be due to free RNA or to other RNA-BPs that bind to the antibody-coupled beads (12). Based on these observations, we infer that sequence libraries made via PAR-CLIP on CJ2192 are expected to be highly enriched for RNAs that are specifically cross-linked by Hfq.

Identification of Hfq-interacting sRNAs using PAR-CLIP

To convert the crosslinked RNA into sequencing libraries, strips containing the Hfqcrosslinked RNA were cut out from nitrocellulose filters and the RNA was released from the 
nitrocellulose strips using proteinase $\mathrm{K}$ to digest proteins. The purified RNAs were converted to sequencing libraries and sequenced on an Illumina HiSeq sequencing system. To assess any contribution from background RNA, PAR-CLIP was similarly performed on RNA derived from CJ2109 that was purified from the same region of the nitrocellulose filters as for FLAG-tagged Hfq. The resulting data were analyzed using the Galaxy bioinformatics platform (13).

We first assessed the fraction of reads that correspond to different classes of RNAs. A comparison of sequence reads between the two libraries revealed that the fraction of reads corresponding to tRNAs and rRNAs did not change appreciably between the two samples, but reads corresponding to non-coding RNAs (ncRNAs) were increased by over two-fold in the library from CJ2192 whereas mRNA reads were decreased (Fig. 3A). These changes are consistent the significant role of $\mathrm{Hfq}$ in interacting with sRNAs, which are included in the ncRNA category, and in targeting mRNAs for translational repression or degradation (14). Next, we assessed the frequency of $\mathrm{T}$ to $\mathrm{C}$ conversions, diagnostic of protein-4SU crosslinking, within the individual sRNAs. We found that 22 sRNAs showed significantly greater $\mathrm{T}>\mathrm{C}$ conversion ratios in the library prepared from CJ2192, as compared to the control library (Fig. 3B). The majority of the sRNAs on this list have been identified as mRNA regulators (15), consistent with the role of $\mathrm{Hfq}$ as an effector of sRNA-mRNA interactions. To further define the effects of Hfq on these sRNAs, we performed RNA-seq on wild-type (wt) and $\Delta h f q$ strains (Supplementary Table 1). Among the sRNAs that were found to display enhanced $\mathrm{T}>\mathrm{C}$ conversion ratios in the Hfqtagged strain, we were able to quantify thirteen (Fig. 3C), half of which were found to be present at a lower level in the $\Delta h f q$ strain, which is consistent with Hfq being a stabilizing factor for many sRNAs (16).

To define specific sites of interaction between Hfq and the sRNAs, we characterized $\mathrm{T}>\mathrm{C}$ conversion ratios at the nucleotide level for each of the 22 sRNAs (Supplementary Table 2). We found the different sRNAs to contain between 1-11 positions that displayed enhanced 
$\mathrm{T}>\mathrm{C}$ ratios, some examples of which are presented in Fig. 3D-G. For the OxyS sRNA, seven sites with increased $\mathrm{T}>\mathrm{C}$ conversion ratios were identified, of which one, at position 68 , showed a two to three-fold higher conversion ratio compared to the other positions (Fig. 3D). Consistent with the increased $\mathrm{T}>\mathrm{C}$ conversion frequency observed at this position, it has been shown that Hfq binding to OxyS is mediated by residues near nt 70 (17). A second sRNA, DsrA, exhibited four positions with enhanced $\mathrm{T}>\mathrm{C}$ conversion ratios (Fig. 3E), of which one, present at the base of the second stem-loop motif in DsrA, exhibited a three-fold higher ratio than the other sites, which suggests that Hfq might be binding to DsrA primarily through recognition of this stem-loop structure. Of significance, none of the six contiguous U-residues preceding this stem-loop exhibited $\mathrm{T}>\mathrm{C}$ conversion changes, which is surprising since stretches of $U$ residues have been identified as an Hfq-binding motif in several other RNAs (11). However, a lack of T>C conversions at these positions is fully consistent with a previous study that showed that mutation of this U-rich stretch has little effect on the interactions between Hfq and DsrA (18). A third sRNA, DicF, was found to exhibit increased $\mathrm{T}>\mathrm{C}$ conversion frequencies only within a single-stranded region, whereas in the final example, a single position within the loop region of OmrB was identified (Figs. 3F and G). Based on the different contexts in which these sites are located on the sRNAs, it indicates that Hfq can bind to RNA within a variety of different RNA motifs.

Identification of Hfq-regulated proteins

The RNA-Seq analysis also identified a number of mRNAs that were differentially expressed in $\Delta h f q$ strains (Supplementary Table 1). However, mRNAs that are translationally regulated by Hfq would not be expected to be identified in this population. To identify additional Hfq-regulated genes, we performed quantitative mass spectrometry analysis on cell extracts derived from wt and $\Delta h f q$ strains. With a view to identify Hfq-regulated genes under more than 
one condition, mass spectrometry was performed both on cells grown to mid-log phase and on cells growth to stationary phase (Supplementary Table 3), as the latter condition is known to increase the extent of regulatory control by Hfq through an increase in the concentration of Hfq and that of several sRNAs via the stabilization role of Hfq (19).

For cells grown to midlog phase, these analyses yielded a quantitation of 1561 proteins. Among these proteins, 35 showed a two-fold or greater increase in the $\Delta h f q$ strain, as compared to the wt strain, and 30 showed a two-fold or greater decrease (Fig. 4A). Similarly, for cells grown to saturated phase, 1641 proteins were quantified of which 45 were upregulated by twofold or more in the $\Delta h f q$ strain, and 75 were downregulated by two-fold or more (Fig. 4B). A comparison of proteins that were upregulated under the two growth conditions a yielded significant overlap (Fig. 4C), but however, more than half of the proteins that were upregulated by two-fold or greater in stationary phase (24/45) were not upregulated to the same extent in mid-log phase. In a similar manner, the majority of proteins downregulated by two-fold or more in midlog phase were also downregulated during stationary phase, but the reverse was not true (Fig. 4D). These data suggest that the effects of $\mathrm{Hfq}$ on gene regulation are generally more significant during stationary phase. Consistent with this notion, among the proteins that were upor down-regulated in both the midlog and stationary phase, a majority (28/42) showed a greater fold-effect in stationary phase (Supplementary Table 3). Nonetheless, we note that several proteins were significantly regulated in the midlog phase, but not in stationary phase.

We next investigated whether the regulated proteins were associated with increase $T>C$ conversion ratios within their mRNAs. Among the 143 proteins that were up-or down-regulated in either growth condition, 21 could be linked to increased $\mathrm{T}>\mathrm{C}$ conversion ratios within their mRNA 5' UTR, coding region and/or the 3' UTRs (Fig. 4E, Supplementary Table 4). An incomplete match between the number of regulated proteins and those exhibiting increased $\mathrm{T}>\mathrm{C}$ conversion frequencies within their mRNAs could be due either to some genes being 
regulated by $\mathrm{Hfq}$ indirectly or because some of the regulated mRNAs are present at a low abundance. We note that the PAR-CLIP libraries were prepared from cells in the midlog phase, whereas most of the proteins that were regulated were identified in stationary phase. To test the latter hypothesis, the 21 mRNAs with increased $\mathrm{T}>\mathrm{C}$ conversion ratios were ranked based on their abundance in the RNA-Seq library. We found that the majority of RNAs were expressed at relatively high levels (Fig. 4F). Therefore, we expect that a significant fraction of the genes that are regulated by $\mathrm{Hfq}$ but could not be associated with $\mathrm{T}>\mathrm{C}$ conversions might yet be direct targets whose low expression precluded the detection of $\mathrm{T}>\mathrm{C}$ events.

Validation of direct interactions between Hfq and Hfq-regulated RNAs

With the identification of several cross-linked mRNAs whose products were also differentially expressed in the $\Delta h f q$ strain, a key question that arose was whether the interaction sites identified by cross-linking accurately define the basis for regulation by Hfq. To address that question, we constructed lacZ reporter fusions with several of the regulated genes to test whether mutations at the cross-linking sites could affect regulation by Hfq. We found that some of the regulated genes, when fused to $l a c Z$, were not appreciably regulated by $\mathrm{Hfq}$ in this context, which might be because some regions important for regulation were absent in these fusions or because the presence of lacZ sequences interferes with Hfq-mediated regulation. Nonetheless, among the fusions that were regulated to a significant degree, several were further analyzed, of which data for three are shown in Figs. 5A-5C, whereas data for additional constructs is summarized in Fig. 5D.

A first fusion that was analyzed was with osmY, which was found to be downregulated at the protein level in the $\Delta h f q$ strain and for which a single PAR-CLIP cross-linking site was identified in the osmY mRNA 5' UTR, 106 nts upstream of the initiation codon. We noticed that 
the cross-linked site was included within a U-rich sequence comprised of five uridine residues within a seven nt tract (Fig. 5A), which could serve as additional sites of Hfq-RNA interactions. To determine whether those residues are responsible for regulation by Hfq, we made an osm $Y$ lacZ mutant construct that converts each of these seven residues to a $\mathrm{C}$ residue. Strikingly, the activity of the wt osmY-lacZ fusion was found to be reduced by 1.5 -fold in the $\Delta h f q$ strain, whereas the effect of $\mathrm{Hfq}$ on the activity of the mutant construct was completely abolished. On the basis of these results, we infer that the cross-linked sites identified by PAR-CLIP define the Hfq binding region on the osmY mRNA.

A second mRNA that was analyzed was mdtK, which was found to cross-link to Hfq 66 nts upstream of the initiation codon, and whose expression was found to be enhanced as adjudged by proteomics analysis. Consistent with the results of these analyses, we found that the expression of a mdtK-lacZ fusion was increased in a $\Delta h f q$ strain by 1.9 -fold. We next mutated nine residues overlapping the cross-linking site and found that the regulation by $\mathrm{Hfq}$ was reduced significantly. These results suggest that the mutated residues largely define the region of the mRNA that is contacted by Hfq. However, since regulation was not completely abolished, some residues outside of the mutated region might also contribute to Hfq binding. Interestingly, we also noted that the mutant $m d t K$-lacZ fusion was expressed at lower levels in both strains, consistent with a positive effect of Hfq on mdtK expression.

A third RNA tested was $y g d Q$, which was found to be upregulated by RNA-Seq and was found to contain two cross-linking sites, one in the 5' UTR and the other in the coding region. The $y g d Q-l a c Z$ fusion was found to be upregulated by RNA-Seq by nearly three-fold in the $\Delta h f q$ background, but mutations at the two cross-liking sites resulted in significant down-regulation of the Hfq-mediated response. In the additional seven fusions that were tested, mutation of between one to six nts around the cross-linking site reduced the extent of Hfq-mediated regulation in all but two examples (Fig. 5D). 
Overall, the general conclusion is that the cross-linking sites identified by PAR-CLIP not only identify the specific sites of interaction with $\mathrm{Hfq}$, but also to define the regions through which Hfq mediates its biological effects with high accuracy. To the best of our knowledge, a rigorous test of whether the contact sites identified via cross-linking methods pinpoint the sites responsible for RNA regulation has not been yet tested. These analysis, therefore, provides a strong validation for the use of PAR-CLIP to identify functionally relevant interaction sites. In summation, the use of PAR-CLIP appears to have high utility both to identify the cross-linking sites of RNA-BPs on a transcriptome-wide scale and the specific regions on the RNAs through which they mediate their regulatory effects.

\section{Discussion}

PAR-CLIP was developed 10 years ago, but within a relatively short time, it has been implemented for use both in higher and lower eukaryotes (20). For reasons that are unclear, this technique has not been used in prokaryotes as yet. To determine whether PAR-CLIP can be used to characterize RNA binding by prokaryotic RNA-BPs, several experiments were performed. First, we found that the addition of $4 S U$ to the growth medium results in the incorporation of this nucleoside into RNA. When cells were grown with $300 \mu \mathrm{M}$ of $4 \mathrm{SU}$ in the medium, the fraction of $4 \mathrm{SU}$ containing residues incorporated into RNA was found to be $1.3 \%$ (Fig. 1), close to the $1.4-2.4 \%$ range of $4 \mathrm{SU}$ incorporation that has been reported for eukaryotic cells (8). Thus, the ability to apply PAR-CLIP in bacteria is not limited by the import of 4 SU into cells or by discrimination against this base by prokaryotic RNA polymerase.

Next, to determine conditions suitable for implementing PAR-CLIP, we evaluated crosslinking efficiency in cells grown with different levels of $4 \mathrm{SU}$. We found that the extent of crosslinking initially rose with increased 4SU concentration, but high 4SU concentrations caused a signal reduction (Fig. 2A). Concurrently, high 4SU concentrations were found to reduce the 
growth rate (Fig. 2E). The highest amount of crosslinking was observed with $500 \mu \mathrm{M} 4 \mathrm{SU}$ in the medium, which therefore represents a concentration suitable for maximum crosslinking efficiency. Alternatively, if minimizing the effects of 4SU on cell growth is desired, PAR-CLIP can also be effectively performed on cells grown with $250 \mu \mathrm{M} 4 \mathrm{SU}$. We expect that a similar approaches will be useful to determine the optimum conditions for using PAR-CLIP in other prokaryotic organisms.

We next applied PAR-CLIP to a strain containing FLAG-tagged Hfq as well to a control untagged strain. A comparison between reads from the two strains showed that the ncRNA category exhibited the largest increase in $T>C$ conversion ratios in the former strain (Fig $3 A$ ), affirming the central role of $\mathrm{Hfq}$ in interactions with sRNAs, with a corresponding decrease in mRNA reads. Furthermore, of the 22 sRNAs enriched in the Hfq libraries (Fig. 3B), only three (SroH, PsrD and SymR) are not established Hfq targets, suggesting a low level of falsepositives regardless of whether these sRNAs are found to be Hfq targets in the future or not. Thus, the use of PAR-CLIP provides confidence that the application of this method to other proteins will yield a substantially reliable list of genuine RNA targets.

A specific advantage of PAR-CLIP over other CLIP methods is that it allows the identification of cross-linking sites at nucleotide resolution through the identification of diagnostic T>C conversion events. Using this criterion, we were able to identify one or more sites associated with an increased $\mathrm{T}>\mathrm{C}$ conversion frequency in several sRNAs and mRNAs. Additionally, by RNA-Seq and proteomics analyses, we identified a large number of genes that were regulated by $\mathrm{Hfq}$ in midlog or stationary phase (Fig. 4; Supplementary Tables 1 and 3). For both the up-regulated and down-regulated genes, cross-linking sites in their mRNA UTRs or coding regions could be identified. Furthermore, for several of these mRNAs, no associated regulatory sRNAs have been identified. These observations suggest that $\mathrm{Hfq}$ may regulate gene expression through a variety of mechanisms that will require further investigation. 
We further tested whether mutation of residues near the cross-linking site impacts regulation, and in almost every case we found that the extent of regulation by Hfq was significantly reduced, indicating the PAR-CLIP accurately identifies the cis-acting RNA sequences through which Hfq regulates the RNAs. Based on these results, we believe that the use of PAR-CLIP has the potential to yield detailed insights into the basis of mRNA regulation by RNA-BPs at a whole-genome level with single-nucleotide resolution.

\section{Methods}

Strains and plasmids: The wild-type strain used for this study is CJ2109, a derivative of MG1655 that contains a point mutation in rph, which restores the reading frame of this prematurely terminated gene (21). Plasmid pCJ1099 was constructed by amplifying chromosomal DNA using primers with the sequences 5'AAAAGCTTGTCATGGCAGGATTATTCATCG-3'

\section{TTGGATCCTACTTGTCATCGTCATCCTTGTAGTCGATGTCATGATCTTTATAATCACCGTCA}

TGGTCTTTGTAGTCCTGCGCAGCGGCAGGTTTACGCGG-3'. The PCR product, which contains the RhIE gene and an appended 3X-FLAG-tag sequence, was digested with BamHI and HindIII and subcloned into the BamHI and HindIII-digested arabinose-inducible expression vector, pMPM-A4 (22). CJ2191 was made by transforming pCJ1099 into CJ2109. Strain CJ2192, was constructed by appending a 3X FLAG-tag sequence to the C-terminus of the chromosomal Hfq gene by using recombineering (23). Strain derivatives containing $\Delta h f q, \Delta / a c Z$ or $\Delta p c n B$ mutations were made by transduction of the respective alleles from the Keio strains collection into CJ2109 (24). LacZ fusion constructs were made by amplifying genes by PCR using E. coli DNA, digesting the amplified products with Bg/2 and EcoR1 or Mfel, and subcloning the products into pLACZY1a digested with BamH1 and EcoR1 (25). The sequence 
of the oligonucleotides used for PCR amplification are shown in Supplementary Table 5. Mutations into these plasmids were introduced using a Q5 site-directed mutagenesis kit (New England Biolabs).

Analysis of 4SU incorporation into RNA: CJ2109 was grown in LB medium in the presence or absence of $300 \mu \mathrm{M} 4 \mathrm{SU}$ and total RNA was isolated from mid-log cells using the hot-phenol method. $15 \mu \mathrm{g}$ of each RNA sample was digested to nucleosides using snake venom phosphodiesterase and alkaline phosphatase, and analyzed by HPLC, as described (8).

PAR-CLIP: $25 \mathrm{ml}$ of bacterial culture was grown to an $\mathrm{OD}_{600}$ of 0.5 in the absence or presence of 4SU added at early log-phase. For strains using CJ2191, cells were grown in LB medium supplemented with ampicillin, and arabinose was added to a final concentration of $0.2 \%$ at $\mathrm{OD}_{600}=0.2$. Cells were cooled rapidly by adding ice and pelleted in a centrifuge. The cell pellets were resuspended in $10 \mathrm{ml}$ of ice-cold saline solution and crosslinked at $365 \mathrm{~nm}$ on ice for five min, or the indicated times, using a CL-1000 ultraviolet crosslinker (UVP). The cells were repelleted by centrifugation and stored at $-80^{\circ} \mathrm{C}$. For cells grown in RDM, cells were harvested at mid-log phase and crosslinked directly at $365 \mathrm{~nm}$ for five minutes $\left(1.7 \mathrm{w} / \mathrm{cm}^{2}\right)$. After crosslinking, the cells were transferred to cold $40 \mathrm{ml}$ tubes containing $10 \mathrm{~g}$ of ice, pelleted by centrifugation and stored at $-80^{\circ} \mathrm{C}$. Thawed cells were resuspended in $200 \mu \mathrm{l} \mathrm{NP-T}$ buffer (50 mM Sodium phosphate, $300 \mathrm{mM} \mathrm{NaCl}, 0.05 \%$ Tween, $\mathrm{pH} 8.0$ ), sonicated and centrifuged in a microfuge at $4^{\circ} \mathrm{C}$. The supernatant fraction was transferred to a new tube and an equal volume of NP-T buffer supplemented with $8 \mathrm{M}$ urea was added. The supernatant was incubated at $65^{\circ} \mathrm{C}$ for five min, followed by the addition of additional NP-T buffer to yield a final urea concentration of $1 \mathrm{M}$. The lysate was added to pre-washed anti-FLAG magnetic beads (15 $\mu$ l of a 50\% bead suspension; Sigma, cat \# A2220) and the mixture was mixed with rotation for $1 \mathrm{~h}$ at 
$4^{\circ} \mathrm{C}$. The beads were collected by centrifugation at $8000 \times g$ for $30 \mathrm{sec}$, resuspended in $100 \mu \mathrm{l}$ NP-T buffer, and washed twice with $100 \mu$ high-salt buffer $\left(50 \mathrm{mM} \mathrm{NaH}_{2} \mathrm{PO}_{4}, 1 \mathrm{M} \mathrm{NaCl}, 0.05 \%\right.$ Tween, $\mathrm{pH}$ 8.0) and twice with $100 \mu \mathrm{l}$ NP-T buffer. The beads were resuspended in $100 \mu \mathrm{l}$ of NP-T buffer containing $1 \mathrm{mM} \mathrm{MgCl} 2$ and 50 units of Benzonase (Sigma; cat \# E1014) and incubated for $10 \mathrm{~min}$ at $37^{\circ} \mathrm{C}$ in a heat block. The beads were washed once with high-salt NP-T buffer, twice with CIP buffer (100 mM NaCl, $50 \mathrm{mM}$ Tris- $\mathrm{HCl} \mathrm{pH} 7.4,10 \mathrm{mM} \mathrm{MgCl}_{2}$ ), resuspended in $100 \mu \mathrm{l}$ CIP buffer with 10 units of calf intestinal alkaline phosphatase (NEB) and incubated for $30 \mathrm{~min}$ at $37^{\circ} \mathrm{C}$ in a heat block. The beads were washed once with high-salt buffer, twice with PNK buffer (50 mM Tris- $\mathrm{HCl}, \mathrm{pH}$ 7.4, $10 \mathrm{mM} \mathrm{MgCl}, 0.1 \mathrm{mM}$ spermidine), resuspended in $100 \mu \mathrm{l}$ PNK buffer with 10 units of T4 polynucleotide kinase and $1 \mu \mathrm{Ci}$ of $\gamma_{-}{ }^{32} \mathrm{P}-\mathrm{ATP}$, and incubated for $30 \mathrm{~min}$ at $37^{\circ} \mathrm{C}$. The beads were washed thrice with NP-T buffer and resuspended in $20 \mu \mathrm{l}$ Protein Loading buffer $(62 \mathrm{mM}$ Tris, $\mathrm{pH}$ 6.8, 2\% SDS, 0.01\% bromophenol blue, $10 \%$ glycerol) and incubated for $3 \mathrm{~min}$ at $95^{\circ} \mathrm{C}$. The beads were pelleted by centrifugation, and the supernatant was loaded on a mini-Protean 4-15\% SDS-polyacrylamide gel (Bio-Rad), along with pre-stained protein markers. The RNA-protein complexes were transferred to nitrocellulose membrane using a Mini Trans-blot electrophoretic transfer cell (BioRad). The protein marker bands were overlaid with radioactive ink, and the membrane was exposed overnight on a phosphorimager screen. The bands corresponding to labeled RNAprotein complexes were cut into small pieces and incubated at $37^{\circ} \mathrm{C}$ with $400 \mu \mathrm{l}$ PK solution [50 mM Tris- $\mathrm{HCl}, \mathrm{pH}$ 7.4, $75 \mathrm{mM} \mathrm{NaCl}, 6 \mathrm{mM}$ EDTA, 1\% SDS, 10 units of RNasin (Promega) and $400 \mu \mathrm{g}$ of proteinase $\mathrm{K}$ (ThermoScientific)] for $30 \mathrm{~min}$ with shaking at $37^{\circ} \mathrm{C} .100 \mu \mathrm{l}$ of $9 \mathrm{M}$ urea solution was added and the incubation was continued for an additional $30 \mathrm{~min} .450 \mu \mathrm{l}$ of the PK/urea solution was mixed with $450 \mu \mathrm{l}$ phenol/chloroform/isoamyl alcohol (25:24:1) alcohol and centrifuged for $12 \min (16,000 \times g)$ at $4^{\circ} \mathrm{C}$. The aqueous phase was precipitated by overnight incubation at $-20^{\circ} \mathrm{C}$ after the addition of 3 volumes of ethanol, $1 / 10$ volume of $3 \mathrm{M}$ 
Sodium Acetate (pH 5.2) and $1 \mu$ of GlycoBlue (Life Technologies). The precipitate was pelleted by centrifugation $\left(30 \mathrm{~min}, 16,000 \times \mathrm{g}, 4^{\circ} \mathrm{C}\right.$ ), washed with $80 \%$ ethanol, centrifuged again (15 $\left.\min , 16,000 \times g, 4^{\circ} \mathrm{C}\right)$, dried and resuspended in $10 \mu \mathrm{l}$ of sterile water.

Sequence library preparation: High throughput sequencing libraries were prepared using RNA isolated from total RNA or PAR-CLIP samples using the NEBNext Multiplex Small RNA Library Prep Set for Illumina (Set 2; cat\# E7580, New England Biolabs) according to the manufacturer's instructions. To prepare RNA-Seq libraries, $1 \mu \mathrm{g}$ of total RNA was digested with 0.01 units of Benzonase for $10 \mathrm{~min}$ at $37^{\circ} \mathrm{C}$ in $20 \mu \mathrm{l}$ of NP-T buffer, followed by extraction with phenol/chloroform/isoamyl alcohol and ethanol precipitation. $100 \mathrm{ng}$ of the digested RNA was used for sequence library preparation as described above. To prepare PAR-CLIP libraries, RNA isolated using the PAR-CLIP protocol was treated similarly.

Bioinformatics Data Analysis: Raw sequencing data were analyzed by using the web-based Galaxy platform (13). Raw data was converted to Fastqsanger format and adaptors were removed using Trim Galore! The reads were aligned to the E. coli genome (Genbank: NC_000913.3) using Bowtie 2. For the analysis of RNA-seq data, uniquely aligned reads corresponding to individual RNAs were determined using htseq-count. For analysis of PARCLIP data, data from five biological replicates from strains CJ2109 and CJ2192 were pooled. $\mathrm{T}>\mathrm{C}$ conversion frequencies at each genome coordinate were determined by using Naïve Variant Caller and Variant Annotator. Sites of Hfq-mediated cross-linking were assigned based on: (a) $\geq 5$ reads from each library, (b) $>0.1$ conversion frequencies for data from strain CJ2192, (b) conversion frequencies in the data for strain CJ2192 $\geq$ twice the conversion frequencies in the data from strain CJ2109. 
Quantitative mass spectrometry: E. coli strains were grown in RDM to mid-log or stationary phase, sonicated and centrifuged. $100 \mu \mathrm{g}$ of proteins from the supernatant fraction were dried under vacuum and processed for digestion using micro S-Traps ${ }^{\mathrm{TM}}$ (Protifi, Huntington, NY) according to the manufacturer's instructions. Briefly, the dried protein was resuspended in $22 \mu \mathrm{l}$ of $5 \%$ SDS, reduced using DTT at $55^{\circ} \mathrm{C}$ for 10 minutes, alkylated using methyl methanethiosulfonate (MMTS) at room temperature for 10 minutes and then spun down at $20 \mathrm{~K}$ for 10 minutes. Subsequently, $2.5 \mu$ of phosphoric acid was added to the sample, followed by $165 \mu \mathrm{l}$ of a mixture of HPLC grade methanol and Protifi binding/wash buffer. Following loading onto the S-Trap ${ }^{\mathrm{TM}}$, samples were washed three times using centrifugation and trypsin was added in $50 \mathrm{mM}$ TEAB at a 1:25 w/w ratio. The S-Trap ${ }^{\mathrm{TM}}$ column was incubated for 1 hour at $47^{\circ} \mathrm{C}$. Following this incubation, $40 \mu \mathrm{l}$ of $50 \mathrm{mM}$ triethylammonium bicarbonate (TEAB) was added to the S-Trap ${ }^{\mathrm{TM}}$ and the peptides were eluted using centrifugation. Elution was repeated once. A third elution using $35 \mu \mathrm{l}$ of $50 \%$ acetonitrile $(A C N)$ was also performed and the eluted peptides were dried under vacuum. Peptides were reconstituted in $50 \mathrm{mM}$ TEAB and their concentrations were determined using the Pierce $^{\mathrm{TM}}$ quantitative fluorometric peptide assay (Thermo Fisher Scientific, Waltham, MA). Twelve micrograms of peptides were labelled with TMT labels (6-plex) according to the manufacturer's instructions (Thermo Fisher Scientific, Waltham, MA) and pooled. The pooled plexed samples were dried under vacuum, resolubilized in 1\% trifluoroacteic acid (TFA), desalted using $2 \mu \mathrm{g}$-capacity C18 ZipTips (Millipore, Billerica, MA), and then dried once again using vacuum. For mass spectrometry, dried TMT-labelled peptides were reconstituted in $5 \mu \mathrm{l}$ of $0.1 \%$ TFA, vortexed briefly, and then sonicated for 15 minutes. The peptides were subsequently on-line eluted into a Fusion Tribrid mass spectrometer (Thermo Scientific, San Jose, CA) from an Acclaim PepMap ${ }^{\text {TM }}$ RSLC C18 nano Viper analytical column $(2 \mu \mathrm{m}, 100 \AA \AA, 75-\mu \mathrm{m}$ ID $\times 50 \mathrm{~cm}$, Thermo Scientific, San Jose, CA $)$ using a gradient of $5-25 \%$ solvent $\mathrm{B}(80 / 20 \mathrm{ACN} /$ water, $0.1 \%$ formic acid) in $180 \mathrm{~min}$, followed by 25 - 
$44 \%$ solvent $B$ in $60 \mathrm{~min}, 44-80 \%$ solvent $B$ in $0.1 \mathrm{~min}$, a 5 min hold of $80 \%$ solvent $B$, a return to $5 \%$ solvent $B$ in $0.1 \mathrm{~min}$, and finally a $20 \mathrm{~min}$ hold of solvent $\mathrm{B}$. All flow rates were $250 \mathrm{nl} / \mathrm{min}$ delivered using a nEasy-LC1000 nano liquid chromatography system (Thermo Scientific, San Jose, CA). Solvent A consisted of water and $0.1 \%$ formic acid. lons were created at $1.7 \mathrm{kV}$ using the EASY-Spray ${ }^{\mathrm{TM}}$ ion source held at $50^{\circ} \mathrm{C}$ (Thermo Scientific, San Jose, CA). A synchronous precursor selection (SPS) $-\mathrm{MS}^{3}$ mass spectrometry method was used, as described (26), scanning between $380-2000 \mathrm{~m} / \mathrm{z}$ at a resolution of 120,000 for MS1 in the Orbitrap mass analyzer, and performing collision-induced dissociation (CID) at top speed in the linear ion trap of peptide monoisotopic ions with charge $2-8$, using a quadrupole isolation of 0.7 $\mathrm{m} / \mathrm{z}$ and a CID energy of $35 \%$. The top $10 \mathrm{MS}^{2}$ ions in the ion trap between $400-1200 \mathrm{~m} / \mathrm{z}$ were then chosen for higher energy collision dissociation (HCD) at 65\% energy and detection in the Orbitrap at a resolution of 60,000 and an automatic gain control (AGC) target of 100,000 and an injection time of $120 \mathrm{msec}\left(\mathrm{MS}^{3}\right)$. Quantitative analysis of the TMT experiments was performed simultaneously to protein identification using Proteome Discoverer 2.3 software (Thermo Scientific, San Jose, CA) using the raw data. The precursor and fragment ion mass tolerances were set to $10 \mathrm{ppm}, 0.6 \mathrm{Da}$, respectively), enzyme was trypsin with a maximum of 2 missed cleavages and the Uniprot Escherichia Coli $\mathrm{K} 12$ proteome FASTA file was used in the SEQUEST searches. The impurity correction factors obtained from Thermo Fisher Scientific for each kit were included in the search and quantification. The following settings were used to search the data: oxidation, +15.995 Da (M); deamidation, +0.984 Da (N, Q); static modifications of TMT 6-plex +229.163 Da (N-terminus K); MMTS +45.988 (C). Only Unique+ Razor peptides were considered for quantification purposes. The Percolator feature of Proteome Discoverer 2.3 was used to set a false discovery rate (FDR) of $1 \%$. Total Peptide Abundance normalization method was used to adjust for loading bias and the protein abundance based method was used to calculate the protein level ratios. Co-isolation threshold and synchronous precursor selection (SPS) mass matches threshold were set to 50 and 65 , respectively. ANOVA was performed by 
individual proteins and the multiple testing correction as per the Benjamini-Hochberg procedure was applied to identify a "top tier" of significant proteins and a column for $p_{\text {adj }}$ values was created.

$\beta$-galactosidase assays: Plasmid constructs were transformed into $\Delta / a c Z$ versions of strains CJ2109 or CJ2109 $\Delta h f q$. In some instances, the strains contained an additional $\Delta p c n B$ mutation, since we showed that the $\triangle p c n B$ mutation reduces the toxicity associated with high levels of lacZ expression (27). Strains were grown in RDM (Teknova) and assayed in mid-log or saturated phase. Each measurement reported is based on four biological replicates.

\section{Acknowledgements}

We thank Dr. Zhongwei Li (Florida Atlantic University) for assistance with HPLC analysis. We thank Thorsten Bischler and Sung-Huan Yu (University of Würzburg) for providing a GFF file with 5' UTR annotations. This work was supported by Grant GM114540 from the National Institutes of Health.

\section{Figure Legends}

Figure. 1. HPLC analysis. Total cellular RNA was isolated from cells grown in the absence (A) or presence of $300 \mu \mathrm{M} 4 \mathrm{SU}(\mathrm{B})$, enzymatically digested to nucleosides, and the products were resolved by HPLC with detection at $260 \mathrm{~nm}$ or $330 \mathrm{~nm}$. Only the regions corresponding to the retention times for uracil $(U)$ and $4 S U$ are shown. For the $260 \mathrm{~nm}$ trace, the mutually overlapping cytosine $(\mathrm{C})$ peak is also included. A background $330 \mathrm{~nm}$ absorbance that is 
present in the RNA sample isolated from cells grown without $4 \mathrm{SU}$ is likely due to $4 \mathrm{SU}$ that is post-transcriptionally incorporated into tRNAs (28).

Figure. 2. PAR-CLIP on FLAG-tagged proteins. (A) CJ2191 was grown in LB supplemented with different concentrations of $4 \mathrm{SU}$ and cells were crosslinked at $365 \mathrm{~nm}$ after harvesting. FLAGtagged RhIE was purified by using anti-FLAG antibodies and the RNAs crosslinked to RhIE were radiolabeled. Proteins were resolved by gel electrophoresis and transferred to nitrocellulose filter. Top, the nitrocellulose filter was exposed to a phosphorimager screen to evaluate the extent of RNA crosslinking to RhIE; bottom, the filter was subsequently analyzed by Western blot using anti-FLAG antibodies. (B) The effect of UV irradiation time on crosslinking efficiency. CJ2191 was grown in LB medium and irradiated at $365 \mathrm{~nm}$ for different periods of time. The crosslinked RhIE was analyzed as in (A). Top, phosphorimager scan; bottom, western-blot analysis. (C) Optical density of LB medium and RDM. The absorbance of LB and RDM was measured at 365 and $254 \mathrm{~nm}$ in the absence or presence of $250 \mu \mathrm{M} 4 \mathrm{SU}$. (D) Effect of washing on cross-linking efficiency. CJ2191 was grown in LB medium containing 250 $\mu \mathrm{M}$ of $4 \mathrm{SU}$ and crosslinked prior to or after washing and resuspension in saline. (E) Effect of 4SU on cell growth. Strain CJ2109 was grown in RDM supplemented with the indicated concentrations of $4 \mathrm{SU}$ and cell doubling times were measured. The indicated standard errors are based on three replicates for each 4SU concentration. (F) PAR-CLIP on single-copy FLAGtagged Hfq. Strains CJ2192 or CJ2109 were grown in RDM containing $250 \mu \mathrm{M}$ of $4 \mathrm{SU}$ and were irradiated after harvest with $365 \mathrm{~nm}$ UV light for five minutes. FLAG-tag protein purification and RNA labeling was performed as in (A).

Figure 3. Analysis of sRNAs. (A) PAR-CLIP on Hfq enriches for ncRNAs. The percentage of uniquely mapping reads from PAR-CLIP libraries from strains CJ2109 (red bars) or CJ2192 
(blue bars), which correspond to different categories of E. coli RNAs, are depicted. (B) sRNAs enriched in Hfq PAR-CLIP libraries. The sRNAs with cumulative $\mathrm{T}>\mathrm{C}$ conversion frequencies that are two-fold or higher in PAR-CLIP libraries from strain CJ2192, relative to strain CJ2109, are listed. \#, T>C changes were observed only in the CJ2192 library. (C) Effect of the $\Delta h f q$ mutation on sRNA abundance. The relative abundance of sRNAs in $\Delta h f q$ and wt strains, as determined by RNA-seq, is listed. (D-F) Sites of enhanced T>C conversions in the OxyS (D), DsrA $(E)$, DicF $(F)$ or OmrB $(G)$ sRNAs. Only sites that displayed a two-fold or greater T>C conversion ratios in libraries made from strain CJ2192, as compared to strain CJ2109, are shown. The $\mathrm{T}>\mathrm{C}$ conversion frequency at these positions is shown in red. The RNAs are depicted based on known structures or computationally derived structures using Mfold (29).

Figure 4. Analysis of hfq-regulated proteins. (A, B), Volcano plot of fold change in protein levels in the $\Delta h f q$ strain vs. P-value for cells grown in RDM to midlog phase (A) or stationary phase (B). P values are based on three replicates each. (C) Overlap of proteins upregulated in the $\Delta h f q$ strain upon growth to midlog phase (red ovals) or stationary phase (blue ovals). (D) Overlap as in (C) for proteins downregulated in the $\Delta h f q$ strain. (E) Location of sites with enhanced $\mathrm{T}>\mathrm{C}$ conversion ratios for mRNAs corresponding to the proteins differentially expressed in the $\Delta h f q$ strain. In one case, (Dps) enhanced T>C conversion sites were identified both in the coding region and in the $3^{\prime}$ UTR of its mRNA, and is included in both categories. (F) Abundance of mRNAs displaying enhanced $\mathrm{T}>\mathrm{C}$ conversion ratios. mRNAs associated with enhanced $\mathrm{T}>\mathrm{C}$ conversion ratios were assigned to one of four quartiles based on RNA-Seq data from a wild-type strain (Supplementary Table 1). Quartile 1 represents the $25 \%$ most abundant mRNAs, and quartile 4, the RNAs that are least abundant. 
Figure 5. Mutation of residues near mRNA cross-linking sites reduces regulation by Hfq. (A-C) Top, sequences of the Hfq-regulated mRNAs for the osmY mRNA (A), mdtK mRNA (B) and ygdQ mRNAs $(\mathrm{C})$. The cross-linked residues are shown in red and the sequences that were mutated depicted by arrows. Bottom, activity of the respective wild-type or mutant lacZ fusions. The lacZ activity of the fusions in wt and $\Delta h f q$ strains is depicted by blue and red bars, respectively. The values above the bars indicate the extent to which the lac $Z$ activity of the fusions is regulated by Hfq. The P-values shown are based on the null hypothesis that mutations in the corresponding construct do not decrease the extent of Hfq-mediated regulation. (D) Analysis of additional Hfq targets identified by PAR-CLIP. The location of the cross-linking site relative to the start of the coding region and the number of residues mutated are indicated. The sequences of the residues near the cross-lining site in the wt and mutant constructs are shown, with the Hfq cross-linked residues in red. The extent of regulation of wt and mutant constructs, and P-values, were calculated as for sections A-C. 
Figure 1

A
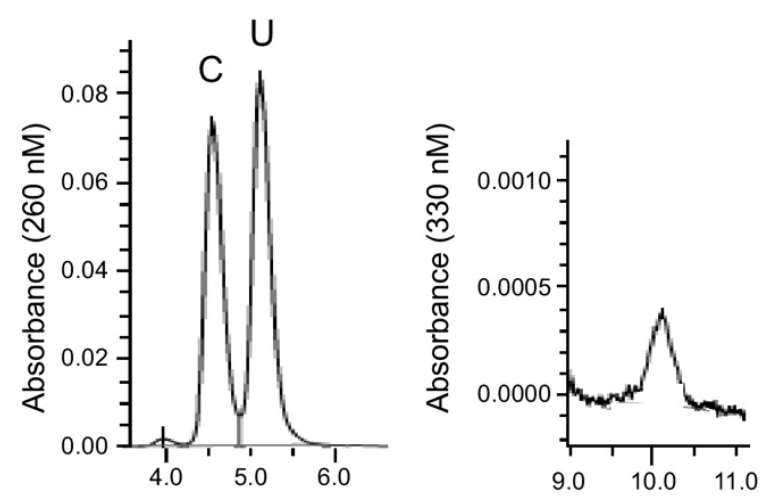

Retention Time (min)
B
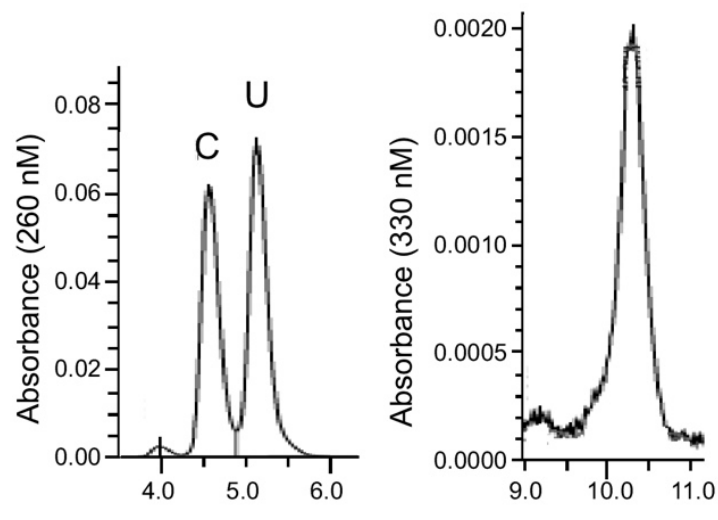

Retention Time (min) 
bioRxiv preprint doi: https://doi.org/10.1101/2020.06.29.178624; this version posted June 30, 2020. The copyright holder for this preprint (which was not certified by peer review) is the author/funder, who has granted bioRxiv a license to display the preprint in perpetuity. It is made available under aCC-BY-NC-ND 4.0 International license.

Figure 2

A

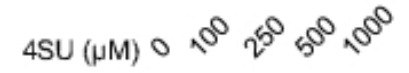

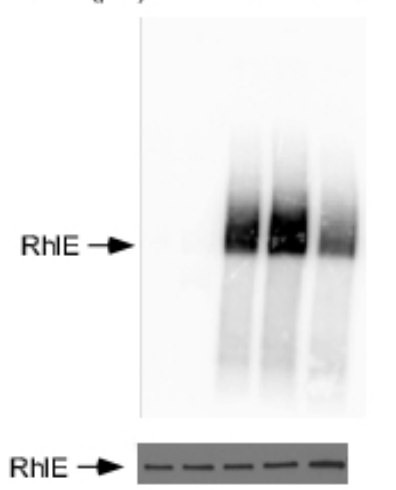

D

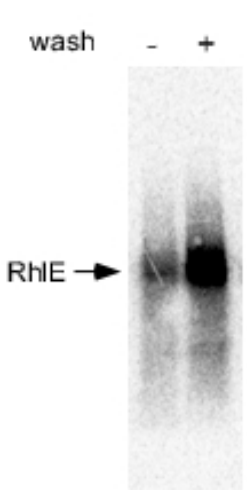

$E$
B

time (min) $00.22^{5} 50$

C
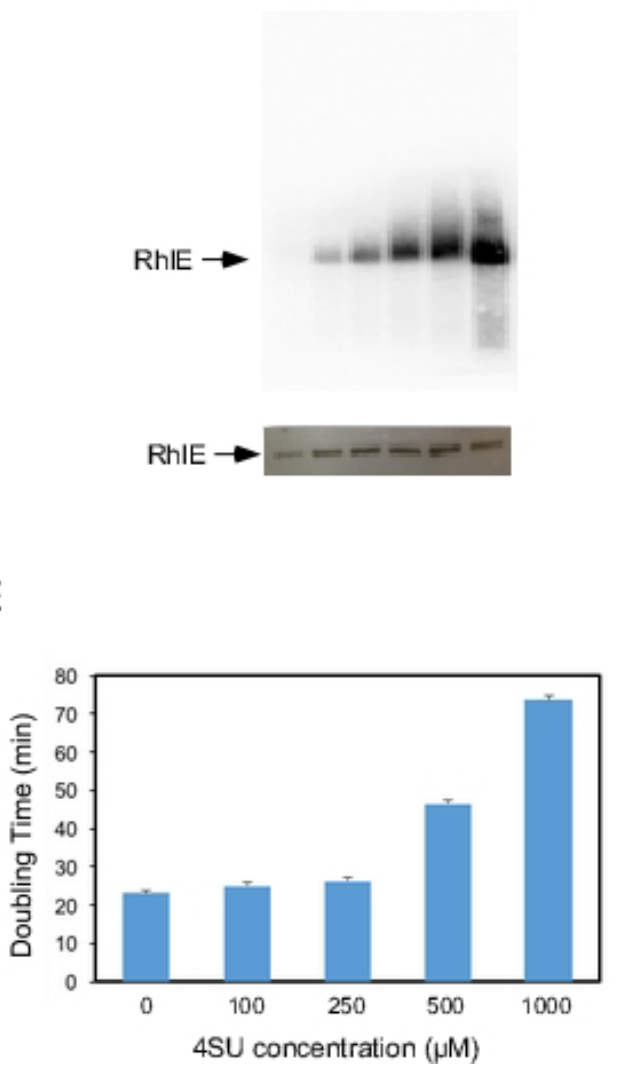

$\mathrm{F}$

$\begin{array}{cccc}\text { wavelength }(\mathrm{nm}) & \text { 4SU } & \text { LB } & \text { RDM } \\ 365 & - & 0.6 & 0.01 \\ 365 & + & 1.1 & 0.5 \\ 254 & - & 20 & 10 \\ 254 & + & 20 & 11 \\ & & & \end{array}$

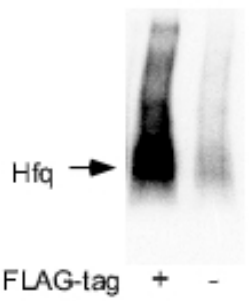


bioRxiv preprint doi: https://doi.org/10.1101/2020.06.29.178624; this version posted June 30, 2020. The copyright holder for this preprint (which was not certified by peer review) is the author/funder, who has granted bioRxiv a license to display the preprint in perpetuity. It is made available under aCC-BY-NC-ND 4.0 International license.

Figure 3

A

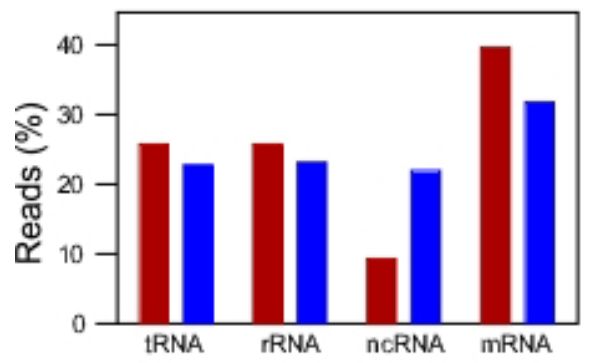

$\mathrm{D}$

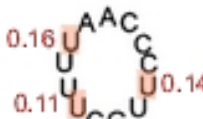

$0.11 \mathrm{CG}^{U}$

$\mathrm{CAA}_{\mathrm{A}}$<smiles>c1ccc2ccccc2c1</smiles>

CG

GC

GC

CG $U$ U

AU 0.15 GC

AU AU 5' GC GA GCG A A UAACUAAAGCCAACGUGAACUUUUGC

F

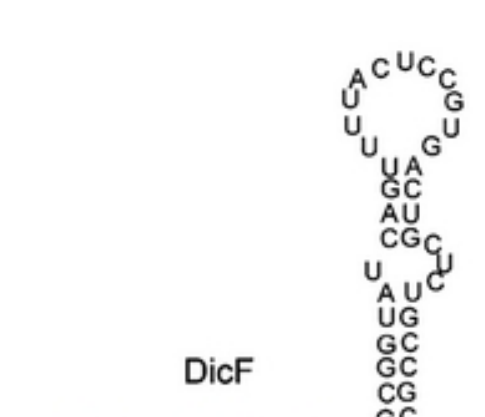

5'UUUCUGGUGACGUUUGC ĞC3'

$\begin{array}{lll}0.22 & 0.14 & 0.21\end{array}$
B

sRNA T>C Ratio (HfqWT)

MicF

Psrd

RydC

SgrS

SroH

GadY

OmrA

RyeA

RyhB

DicF

Mcas

OmrB

ArcZ

MicA

GcvB

SymR

RybB

GImZ

Dsra

Spf

Oxys

Chix
G
C

SRNA

Rel. abundance ( $N h f q / W T)$

ArcZ

0.04

GadY $\quad 0.06$

ChiX $\quad 0.07$

Mcas $\quad 0.09$

DsrA $\quad 0.26$

Spf $\quad 0.38$

Oxys $\quad 0.50$

GimZ $\quad 0.68$

PsrD $\quad 0.76$

RyeA $\quad 0.83$

$\mathrm{SroH} \quad 0.85$

SymR $\quad 0.98$

GevB $\quad 1.95$

E

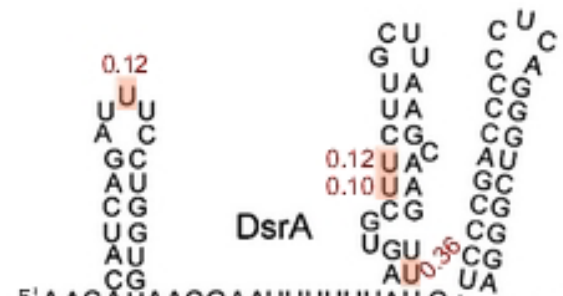

5'AACAUAACGAAUUUUUUUU CAUUU'

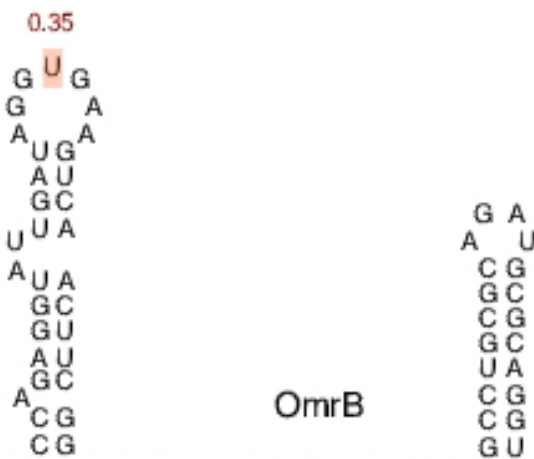

5'C GUUGAGCACAUGA AUUACACCA UUUUUUU' 
bioRxiv preprint doi: https://doi.org/10.1101/2020.06.29.178624; this version posted June 30, 2020. The copyright holder for this preprint (which was not certified by peer review) is the author/funder, who has granted bioRxiv a license to display the preprint in perpetuity. It is made available under aCC-BY-NC-ND 4.0 International license.

Figure 4

A

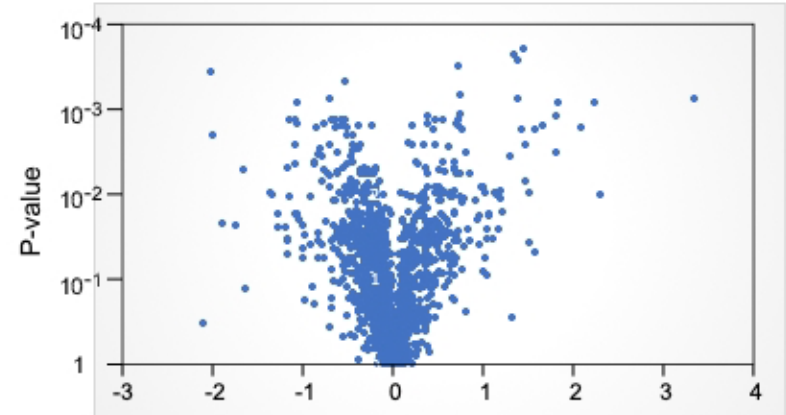

$\log _{2}$ relative abundance $(\Delta h f q / W T)$
B

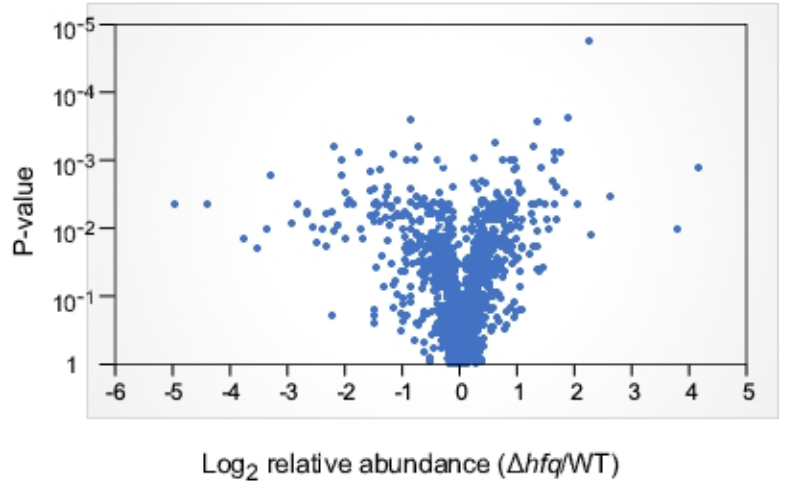

C
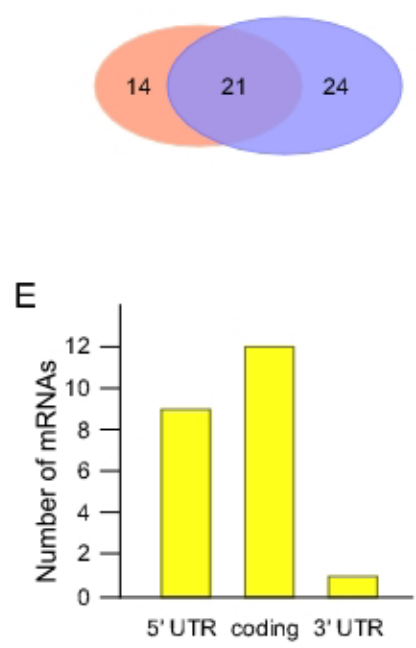

D

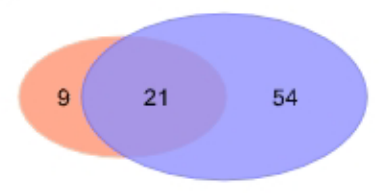

F

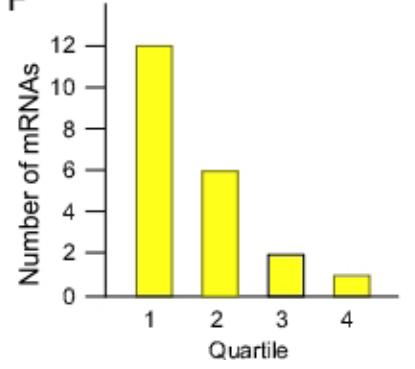


bioRxiv preprint doi: https://doi.org/10.1101/2020.06.29.178624; this version posted June 30, 2020. The copyright holder for this preprint (which was not certified by peer review) is the author/funder, who has granted bioRxiv a license to display the preprint in perpetuity. It is made available under aCC-BY-NC-ND 4.0 International license.

Figure 5

A
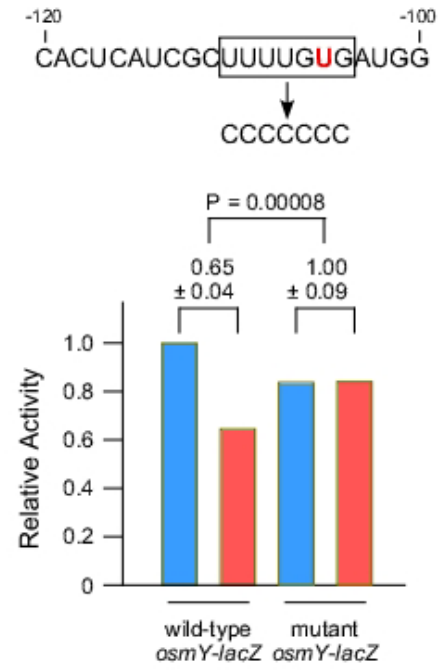

$\mathrm{D}$

$\begin{array}{ccc}\text { Reporter Fusion } & \begin{array}{c}\text { Cross-linking } \\ \text { site location }\end{array} & \begin{array}{c}\text { \# residues } \\ \text { mutated }\end{array} \\ \text { cycA-lacZ } & +125 & 6 \\ \text { glpF-lacZ } & +232 & 5 \\ \text { cstA-lacZ } & -37 & 5 \\ \text { nfsA-lacZ } & +48 & 4 \\ \text { nlpC-lacZ } & +18 & 4 \\ \text { mog-lacZ } & +9 & 3 \\ \text { fabA-lacZ } & +39 & 1\end{array}$

B
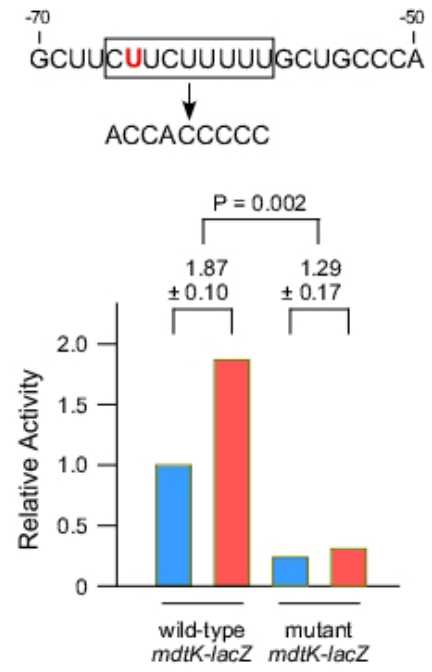

Eold regulation ( $\triangle h f g N T$ strain)

wt sequence mutant sequence

TTGTTT

TGTTT

CCACCC

Significance

AATGT

ATTT

TTTT

TTT

T
C
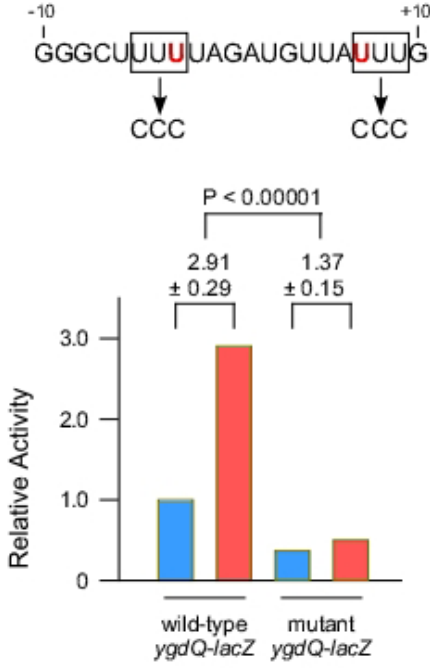

$3.85 \pm 0.19$

$3.27 \pm 0.19$

$3.20 \pm 0.26$

$1.73 \pm 0.03$

$0.85 \pm 0.05$

$0.99 \pm 0.04$

$0.52 \pm 0.02$

C

$0.86 \pm 0.05$

$0.72 \pm 0.06$
$2.17 \pm 0.08$
WT construct Mutant construct

$1.68 \pm 0.13$

$1.02 \pm 0.09$

(P-value)

0.00002

$<0.00001$

0.41

0.00014

$<0.00001$

0.028

0.99 


\section{References}

1. Tenenbaum, S.A., Carson, C.C., Lager, P.J. and Keene, J.D. (2000) Identifying mRNA subsets in messenger ribonucleoprotein complexes by using cDNA arrays. Proceedings of the National Academy of Sciences of the United States of America, 97, 14085-14090.

2. Ule, J., Jensen, K.B., Ruggiu, M., Mele, A., Ule, A. and Darnell, R.B. (2003) CLIP identifies Nova-regulated RNA networks in the brain. Science, 302, 1212-1215.

3. Jensen, K.B. and Darnell, R.B. (2008) CLIP: crosslinking and immunoprecipitation of in vivo RNA targets of RNA-binding proteins. Methods in molecular biology (Clifton, N.J.), 488, 85-98.

4. Singh, G., Ricci, E.P. and Moore, M.J. (2014) RIPiT-Seq: a high-throughput approach for footprinting RNA:protein complexes. Methods (San Diego, Calif.), 65, 320-332.

5. Yeh, H.S., Chang, J.W. and Yong, J. (2016) Ribo-Proteomics Approach to Profile RNAProtein and Protein-Protein Interaction Networks. Methods in molecular biology (Clifton, N.J.), 1421, 165-174.

6. Hafner, M., Landthaler, M., Burger, L., Khorshid, M., Hausser, J., Berninger, P., Rothballer, A., Ascano, M., Jr., Jungkamp, A.C., Munschauer, M. et al. (2010) Transcriptome-wide identification of RNA-binding protein and microRNA target sites by PAR-CLIP. Cell, 141, 129-141.

7. Danan, C., Manickavel, S. and Hafner, M. (2016) PAR-CLIP: A Method for TranscriptomeWide Identification of RNA Binding Protein Interaction Sites. Methods in molecular biology (Clifton, N.J.), 1358, 153-173.

8. Spitzer, J., Hafner, M., Landthaler, M., Ascano, M., Farazi, T., Wardle, G., Nusbaum, J., Khorshid, M., Burger, L., Zavolan, M. et al. (2014) PAR-CLIP (Photoactivatable Ribonucleoside-Enhanced Crosslinking and Immunoprecipitation): a step-by-step protocol to the transcriptome-wide identification of binding sites of RNA-binding proteins. Methods Enzymol, 539, 113-161.

9. Jain, C. (2008) The E. coli RhIE RNA helicase regulates the function of related RNA helicases during ribosome assembly. RNA (New York, N.Y.), 14, 381-389.

10. Faner, M.A. and Feig, A.L. (2013) Identifying and characterizing Hfq-RNA interactions. Methods (San Diego, Calif.), 63, 144-159.

11. Holmqvist, E., Wright, P.R., Li, L., Bischler, T., Barquist, L., Reinhardt, R., Backofen, R. and Vogel, J. (2016) Global RNA recognition patterns of post-transcriptional regulators $\mathrm{Hfq}$ and CsrA revealed by UV crosslinking in vivo. The EMBO journal, 35, 991-1011.

12. Friedersdorf, M.B. and Keene, J.D. (2014) Advancing the functional utility of PAR-CLIP by quantifying background binding to mRNAs and IncRNAs. Genome Biol, 15, R2.

13. Afgan, E., Baker, D., van den Beek, M., Blankenberg, D., Bouvier, D., Cech, M., Chilton, J., Clements, D., Coraor, N., Eberhard, C. et al. (2016) The Galaxy platform for accessible, reproducible and collaborative biomedical analyses: 2016 update. Nucleic acids research, 44, W3-W10.

14. Storz, G., Vogel, J. and Wassarman, K.M. (2011) Regulation by small RNAs in bacteria: expanding frontiers. Molecular cell, 43, 880-891. 
15. Melamed, S., Peer, A., Faigenbaum-Romm, R., Gatt, Y.E., Reiss, N., Bar, A., Altuvia, Y., Argaman, L. and Margalit, H. (2016) Global Mapping of Small RNA-Target Interactions in Bacteria. Molecular cell, 63, 884-897.

16. Schu, D.J., Zhang, A., Gottesman, S. and Storz, G. (2015) Alternative Hfq-sRNA interaction modes dictate alternative mRNA recognition. The EMBO journal, 34, 25572573.

17. Zhang, A., Wassarman, K.M., Ortega, J., Steven, A.C. and Storz, G. (2002) The Sm-like Hfq protein increases OxyS RNA interaction with target mRNAs. Molecular cell, 9, 11-22.

18. Brescia, C.C., Mikulecky, P.J., Feig, A.L. and Sledjeski, D.D. (2003) Identification of the $\mathrm{Hfq}$-binding site on DsrA RNA: Hfq binds without altering DsrA secondary structure. RNA (New York, N.Y.), 9, 33-43.

19. Iosub, I.A., van Nues, R.W., McKellar, S.W., Nieken, K.J., Marchioretto, M., Sy, B., Tree, J.J., Viero, G. and Granneman, S. (2020) Hfq CLASH uncovers sRNA-target interaction networks linked to nutrient availability adaptation. Elife, 9.

20. Yamaji, M., Jishage, M., Meyer, C., Suryawanshi, H., Der, E., Yamaji, M., Garzia, A., Morozov, P., Manickavel, S., McFarland, H.L. et al. (2017) DND1 maintains germline stem cells via recruitment of the CCR4-NOT complex to target mRNAs. Nature, 543, 568572.

21. Gutgsell, N.S. and Jain, C. (2012) Gateway role for rRNA precursors in ribosome assembly. Journal of bacteriology, 194, 6875-6882.

22. Mayer, M.P. (1995) A new set of useful cloning and expression vectors derived from pBlueScript. Gene, 163, 41-46.

23. Ellis, H.M., Yu, D., DiTizio, T. and Court, D.L. (2001) High efficiency mutagenesis, repair, and engineering of chromosomal DNA using single-stranded oligonucleotides. Proceedings of the National Academy of Sciences of the United States of America, 98, 6742-6746.

24. Baba, T., Ara, T., Hasegawa, M., Takai, Y., Okumura, Y., Baba, M., Datsenko, K.A., Tomita, M., Wanner, B.L. and Mori, H. (2006) Construction of Escherichia coli K-12 in-frame, single-gene knockout mutants: the Keio collection. Mol Syst Biol, 2, 20060008.

25. Jain, C. (1993) New improved lacZ gene fusion vectors. Gene, 133, 99-102.

26. Ting, L., Rad, R., Gygi, S.P. and Haas, W. (2011) MS3 eliminates ratio distortion in isobaric multiplexed quantitative proteomics. Nat Methods, 8, 937-940.

27. Ojha, S. and Jain, C. (2020) Dual-level autoregulation of the E. coli DeaD RNA helicase via mRNA stability and Rho-dependent transcription termination. RNA (New York, N.Y.).

28. Mueller, E.G., Buck, C.J., Palenchar, P.M., Barnhart, L.E. and Paulson, J.L. (1998) Identification of a gene involved in the generation of 4-thiouridine in tRNA. Nucleic acids research, 26, 2606-2610.

29. Zuker, M. (2003) Mfold web server for nucleic acid folding and hybridization prediction. Nucleic acids research, 31, 3406-3415. 